\title{
Stability of Sarma phases in density imbalanced electron-hole bilayer systems
}

\author{
A. L. Subası, ${ }^{1, *}$ P. Pieri, ${ }^{2}$ G. Senatore, ${ }^{3,4}$ and B. Tanatar ${ }^{1}$ \\ ${ }^{1}$ Department of Physics, Bilkent University, Bilkent, Ankara 06800, Turkey \\ ${ }^{2}$ Dipartimento di Fisica, Scuola di Scienze e Tecnologie, Università di Camerino, I-62032 Camerino, Italy \\ ${ }^{3}$ CNR-INFM DEMOCRITOS National Simulation Center, Trieste, Italy \\ ${ }^{4}$ Dipartimento di Fisica, Università di Trieste, Strada Costiera 11, I-34151 Trieste, Italy
}

(Received 14 December 2009; published 25 February 2010)

\begin{abstract}
We study excitonic condensation in an electron-hole bilayer system with unequal layer densities at zero temperature. Using mean-field theory we solve the Bardeen-Cooper-Schrieffer (BCS) gap equations numerically and investigate the effects of intralayer interactions. The electron-hole system evolves from BCS in the weak coupling limit to Bose-Einstein condensation (BEC) in the strong coupling limit. We analyze the stability of the Sarma phase with $\mathbf{k},-\mathbf{k}$ pairing by calculating the superfluid mass density and also by checking the compressibility matrix. We find that with bare Coulomb interactions the superfluid density is always positive in the Sarma phase, due to a peculiar momentum structure of the gap function originating from the singular behavior of the Coulomb potential at zero momentum and the presence of a sharp Fermi surface. Introducing a simple model for screening, we find that the superfluid density becomes negative in some regions of the phase diagram, corresponding to an instability toward a Fulde-Ferrel-Larkin-Ovchinnikov-type superfluid phase. Thus, intralayer interaction and screening together can lead to a rich phase diagram in the BCS-BEC crossover regime in electron-hole bilayer systems.
\end{abstract}

DOI: 10.1103/PhysRevB.81.075436

PACS number(s): 73.21.Ac, 03.75.Hh, 03.75.Ss

\section{INTRODUCTION}

Recent advances in the trapping and cooling down to degeneracy of ultracold Fermi gases have revived interest in the ground-state phases of these systems. ${ }^{1-14}$ In a twocomponent Fermi system with equal densities attractive interactions between different species lead to Bardeen-CooperSchrieffer (BCS) pairing in the weak coupling limit and Bose-Einstein condensation (BEC) in the strong coupling limit. ${ }^{15}$ When the densities are imbalanced more exotic phases are expected to follow, such as the Sarma phase ${ }^{16}$ with zero center-of-mass momentum and Fulde-FerrellLarkin-Ovchinnikov (FFLO) phase $\mathrm{e}^{17,18}$ with finite center-ofmass momentum. There is a growing literature on the possible phases of two-component Fermi gases with population and mass imbalance. ${ }^{19-41}$ The experimental efforts in ultracold Fermi gases are in their beginning stage and so far only phase separation between a superfluid and a normal (N) phase has been observed. ${ }^{7,8,42,43}$

Semiconducting electron-hole bilayer systems offer another realization of a two-component Fermi system with which the exotic phases can be studied. Formation of excitons between spatially separated electrons and holes and their subsequent condensation have long been predicted ${ }^{44,45}$ and arguably observed nearly 30 years later experimentally. ${ }^{46}$ The phase diagram of symmetric electron-hole bilayer systems (equal mass and layer density) is most reliably calculated by quantum Monte Carlo simulations. ${ }^{47,48}$ Recent success in fabricating closely spaced semiconducting electronhole bilayer structures 49,50 and the ability to control the densities of individual layers make the investigation of Sarma and FFLO phases very timely. In fact, experiments supporting evidence of a transition from the Fermi-liquid phase to an excitonic condensate have been recently reported through Coulomb drag measurements ${ }^{51,52}$ in such structures.
The BCS-BEC crossover in an electron-hole bilayer system with unequal electron and hole densities was recently studied in Ref. 53 within a BCS mean-field approach. Sarma and FFLO phases were found to be stable in some range of densities; electron-hole bilayers appear thus promising candidates for the detection of such elusive phases.

In this paper we extend the work of Ref. 53 by including the in-plane Coulomb interactions, that were neglected there, as well as some screening effects. We find that the effect of intralayer Fock energy quantitatively changes the phase diagram moving the normal-condensed phase boundary to lower densities. Comparing energy of the condensed phase with that of the normal phase, we map out the phase diagram in the average density-population polarization plane. We check the "local" stability of the Sarma phase with respect to competing FFLO order by calculating the superfluid mass density and identify a negative superfluid mass density with an instability toward an FFLO phase. We calculate also the compressibility matrix in order to investigate possible instabilities toward phase separation.

Finally, we consider the effect of gate layer screening, which proves especially important in the discussion of the local stability of the Sarma phase. At zero temperature, the simultaneous presence of the singularity in the Coulomb potential and of a sharp Fermi surface produces, in fact, a logarithmic divergence in the momentum dependence of the BCS gap function which makes the Sarma phase always locally stable against the FFLO phase. The inclusion of some form of screening removes this peculiar behavior, thus recovering the instability toward the FFLO phase in some region of the phase diagram. The intralayer interactions and screening effects give therefore rise to a rich phase diagram in the crossover region between the BCS-like high-density state and the BEC of low-density excitons, showing the possibility to observe exotic superfluid phases as the population polarization is changed. 
The rest of this paper is organized as follows. In the next section we outline the mean-field theory for electron-hole bilayers and provide the set of self-consistent equations for the quasiparticle energies and gap function. In Sec. III, after a brief remark about our computational procedure, we present our results for the quasiparticle properties and phase diagram of the system. We conclude in Sec. IV with a summary and outlook.

\section{MEAN-FIELD THEORY}

The Hamiltonian describing electrons and holes in a bilayer system interacting with the Coulomb potential can be written as

$$
\begin{aligned}
\hat{H}= & \sum_{\mathbf{k}}\left(\epsilon_{\mathbf{k}}^{a} a_{\mathbf{k}}^{\dagger} a_{\mathbf{k}}+\epsilon_{\mathbf{k}}^{b} b_{\mathbf{k}}^{\dagger} b_{\mathbf{k}}\right)+\frac{1}{2 A} \sum_{\mathbf{k}_{1} \mathbf{k}_{2} \mathbf{q}} U_{\mathbf{q}}^{a a} a_{\mathbf{k}_{1}+\mathbf{q}}^{\dagger} a_{\mathbf{k}_{2}-\mathbf{q}}^{\dagger} a_{\mathbf{k}_{2}} a_{\mathbf{k}_{1}} \\
& +\frac{1}{2 A_{\mathbf{k}_{1} \mathbf{k}_{2} \mathbf{q}}} U_{\mathbf{q}}^{b b} b_{\mathbf{k}_{1}+\mathbf{q}}^{\dagger} b_{\mathbf{k}_{2}-\mathbf{q}}^{\dagger} b_{\mathbf{k}_{2}} b_{\mathbf{k}_{1}} \\
& +\frac{1}{A} \sum_{\mathbf{k}_{1} \mathbf{k}_{2} \mathbf{q}} U_{\mathbf{q}}^{a b} a_{\mathbf{k}_{1}+\mathbf{q}}^{\dagger} b_{\mathbf{k}_{2}-\mathbf{q}}^{\dagger} b_{\mathbf{k}_{2}} a_{\mathbf{k}_{1}} .
\end{aligned}
$$

The basis states for electrons and holes are chosen to be plane-wave states labeled by two-dimensional (2D) wave vectors $\mathbf{k}$ as is conventional for a uniform system. The operators $a_{\mathbf{k}} / a_{\mathbf{k}}^{\dagger}\left(b_{\mathbf{k}} / b_{\mathbf{k}}^{\dagger}\right)$ are creation/annihilation operators for electrons (holes), respectively. The single-particle energies are denoted by $\epsilon_{\mathbf{k}}^{a}, \epsilon_{\mathbf{k}}^{b}$ and the matrix element $U_{\mathbf{q}}$ with respect to plane-wave states becomes the Fourier transform of the corresponding two-body Coulomb interaction $U(\mathbf{r})$

$$
U_{\mathbf{q}}^{a a}=U_{\mathbf{q}}^{b b}=\frac{2 \pi e^{2}}{\varepsilon q}, \quad U_{\mathbf{q}}^{a b}=\frac{2 \pi e^{2}}{\varepsilon q} e^{-q d},
$$

where $U^{a a}, U^{b b}$, and $U^{a b}$ denote the electron-electron, holehole, and electron-hole Coulomb interactions, respectively, $A$ is the area of a layer, and $d$ is the interlayer separation. We disregard the spin degrees of freedom.

The bilayer system is characterized by the electron and hole densities, or equivalently by the average density parameter $r_{s}$ (average distance between particles in the plane in units of Bohr radius $a_{B}$ ), the population polarization $\alpha$ (characterizing population imbalance in terms of the ratio of density difference and total density) defined by

$$
n=\frac{1}{2}\left(n_{a}+n_{b}\right)=\frac{1}{\pi a_{B}^{2} r_{s}^{2}} \quad \text { and } \quad \alpha=\frac{n_{a}-n_{b}}{n_{a}+n_{b}}
$$

and the interlayer separation $d$.

The solution of the mean-field Hamiltonian at zero temperature $(T=0)$ is given by the following coupled integral equations:

$$
\Delta_{\mathbf{k}}=-\frac{1}{A} \sum_{\mathbf{k}^{\prime} \neq \mathbf{k}} U_{\mathbf{k}-\mathbf{k}^{\prime}}^{a b} \frac{\Delta_{\mathbf{k}^{\prime}}}{2 E_{\mathbf{k}^{\prime}}}\left(1-f_{\mathbf{k}^{\prime}}^{+}-f_{\mathbf{k}^{\prime}}^{-}\right),
$$

$$
\begin{gathered}
\xi_{\mathbf{k}}=\epsilon_{\mathbf{k}}-\mu-\frac{1}{2 A} \sum_{\mathbf{k}^{\prime} \neq \mathbf{k}} U_{\mathbf{k}-\mathbf{k}^{\prime}}^{a a}\left[\left(1-\xi_{\mathbf{k}^{\prime}} / E_{\mathbf{k}^{\prime}}\right)\right. \\
\left.\times\left(1-f_{\mathbf{k}^{\prime}}^{+}-f_{\mathbf{k}^{\prime}}^{-}\right)+f_{\mathbf{k}^{\prime}}^{+}+f_{\mathbf{k}^{\prime}}^{-}\right], \\
E_{\mathbf{k}}^{2}=\xi_{\mathbf{k}}^{2}+\Delta_{\mathbf{k}}^{2}, \\
f_{\mathbf{k}}^{ \pm}= \begin{cases}1 & \text { if } E_{\mathbf{k}}^{ \pm}<0 \\
0 & \text { if } E_{\mathbf{k}}^{ \pm}>0,\end{cases}
\end{gathered}
$$

where $\epsilon_{\mathbf{k}}=\left(\epsilon_{\mathbf{k}}^{a}+\epsilon_{\mathbf{k}}^{b}\right) / 2\left(\right.$ with $\left.\epsilon_{\mathbf{k}}^{i}=\hbar^{2} k^{2} / 2 m_{i}, i=a, b\right)$, the mean chemical potential $\mu=\left(\mu_{a}+\mu_{b}\right) / 2$, while

$$
\begin{gathered}
E_{\mathbf{k}}^{ \pm}=E_{\mathbf{k}} \pm \Delta E_{\mathbf{k}}, \\
\Delta E_{\mathbf{k}}=\Delta \xi_{\mathbf{k}}+\frac{1}{2 A} \sum_{\mathbf{k}^{\prime} \neq \mathbf{k}} U_{\mathbf{k}-\mathbf{k}^{\prime}}^{a a}\left(f_{\mathbf{k}^{\prime}}^{-}-f_{\mathbf{k}^{\prime}}^{+}\right), \\
\Delta \xi_{\mathbf{k}}=\frac{1}{2}\left(\epsilon_{\mathbf{k}}^{a}-\mu_{a}-\epsilon_{\mathbf{k}}^{b}+\mu_{b}\right) .
\end{gathered}
$$

At finite temperature, the occupation functions $f_{\mathbf{k}}^{ \pm}\left(E_{\mathbf{k}}^{ \pm}\right)$go from the step function to the Fermi-Dirac distribution.

Given the electron and hole chemical potentials $\mu_{a}$ and $\mu_{b}$, these equations can be solved numerically to obtain the unknown functions $\Delta_{\mathbf{k}}, \xi_{\mathbf{k}}$, and $\Delta E_{\mathbf{k}}$. Physically, $\Delta_{\mathbf{k}}$ is the BCS (s-wave) gap function while $E_{\mathbf{k}}^{ \pm}$are the quasiparticle excitation energies in the superfluid phase. In the absence of intralayer interaction $\xi_{\mathbf{k}}$ is just the average of the free electron and hole dispersions (with respect to the corresponding chemical potentials). Intralayer interaction modifies the free dispersions by the inclusion of the exchange (Fock) interaction, as explicitly considered in Eq. (5).

For fixed number of particles the chemical potential values are adjusted to satisfy the number equations

$$
n_{a}=\frac{1}{2 A} \sum_{\mathbf{k}}\left[\left(1+\frac{\xi_{\mathbf{k}}}{E_{\mathbf{k}}}\right) f_{\mathbf{k}}^{+}+\left(1-\frac{\xi_{\mathbf{k}}}{E_{\mathbf{k}}}\right)\left(1-f_{\mathbf{k}}^{-}\right)\right]
$$

and

$$
n_{b}=\frac{1}{2 A} \sum_{\mathbf{k}}\left[\left(1+\frac{\xi_{\mathbf{k}}}{E_{\mathbf{k}}}\right) f_{\mathbf{k}}^{-}+\left(1-\frac{\xi_{\mathbf{k}}}{E_{\mathbf{k}}}\right)\left(1-f_{\mathbf{k}}^{+}\right)\right] .
$$

In the above mean-field description of the electron-hole bilayer we have used the bare Coulomb interaction given in Eq. (2). In realistic systems, the interactions entering the model hamiltonian of Eq. (1) should be modified to include many-body effects such as exchange and correlation and external potentials. These effects are described by a screening function which usually decreases the strength of the bare Coulomb interaction for electrons and holes in the normal phase. However, the 2D screening due to intralayer and interlayer interactions is difficult to take into account properly for the condensed phase. ${ }^{54}$ In order to see the qualitative effects of screening we consider the mechanism of gate screening which can be taken into account in a simple way. In this mechanism the Coulomb potential of a point charge is replaced by that of a dipole consisting of the point charge 
and its image behind the metallic gate. We have approximately modeled the screening by the gate potential by taking the intralayer and interlayer interactions to be

$$
U_{\mathbf{q}}^{a a}=U_{\mathbf{q}}^{b b}=\frac{2 \pi e^{2}}{\varepsilon \sqrt{q^{2}+\kappa^{2}}}, \quad U_{\mathbf{q}}^{a b}=\frac{2 \pi e^{2}}{\varepsilon \sqrt{q^{2}+\kappa^{2}}} e^{-q d},
$$

respectively, where the parameter $\kappa$ is a screening wave number. In recent experiments with metallic gates to control the charge densities, the separation between the gate and 2D layer is about $\sim 250 \mathrm{~nm} .^{50,55}$ Thus, the image charge is $\sim 500 \mathrm{~nm}$ away from the real charge and we may assume that for distances larger than $500 \mathrm{~nm}$, the Coulomb potential will be screened. In our calculations we take the screening length associated with gate screening to be $\sim 20 a_{B}$, i.e., $\kappa$ $=1 / 20 a_{B}$. We have also checked other values of $\kappa$ and found that the results are largely insensitive in the range 40 $>1 /\left(\kappa a_{B}\right)>5$.

\section{RESULTS AND DISCUSSION}

\section{A. Numerical procedure}

We solve the gap equations by representing the unknown functions on a grid of $k$ points (after angular integration) and using a nonlinear root finding scheme for the function values on grid points. For balanced populations an iterative scheme provides a robust method of solution. For imbalanced populations we employ a root finding scheme for the function values on grid points and chemical-potential values. We start with the equal density solution at the same average density and create imbalance first at a small finite temperature and then decrease the temperature (using the solution from the previous step as input) until results do not change with temperature any more. The integrals are evaluated using Gaussian quadrature. The finite temperature is necessary to obtain smooth functions and gradients for the Newton-Raphson root finding algorithm. We found it necessary to introduce up to three different grids for integration to handle "discontinuities" at low temperatures, when one type of occupation number becomes equal to unity $f_{\mathbf{k}}^{\lambda}=1(\lambda=+$ or -$)$ in a region of $k$ space (and causing the integrands to vanish there).

The so-called Sarma states obtained in this way are of the following BCS form:

$$
|\Psi\rangle=\prod_{\mathbf{q} \in \mathcal{R}} a_{\mathbf{q}}^{\dagger} \prod_{\mathbf{k} \notin \mathcal{R}}\left(u_{\mathbf{k}}+v_{\mathbf{k}} a_{\mathbf{k}}^{\dagger} b_{-\mathbf{k}}^{\dagger}\right)|0\rangle,
$$

where the resulting wave function has a certain range of $\mathbf{k}$ states (the set denoted by $\mathcal{R}$ ) occupied with quasiparticles of the BCS theory giving rise to population imbalance. The region $\mathcal{R}$ is where the quasiparticle energy $E_{\mathbf{k}}^{ \pm}$becomes negative, i.e., less than that of the ground pair energy and the corresponding quasiparticle occupation becomes unity. Incidentally, the quasiparticles of BCS theory are just electron or hole states at that wave vector $\mathbf{k}$. Outside the set $\mathcal{R}$ we have pairs $\mathbf{k},-\mathbf{k}$ of electrons and holes. ${ }^{56}$ Therefore, at $T=0$ there can be one or two Fermi surfaces depending where the set of $\mathbf{k} \in \mathcal{R}$ vectors are. These topologically different phases will be called Sarma-1 (S1) and Sarma-2 (S2). These states have also been called breached pair states ${ }^{22}$ displaying a Fermi
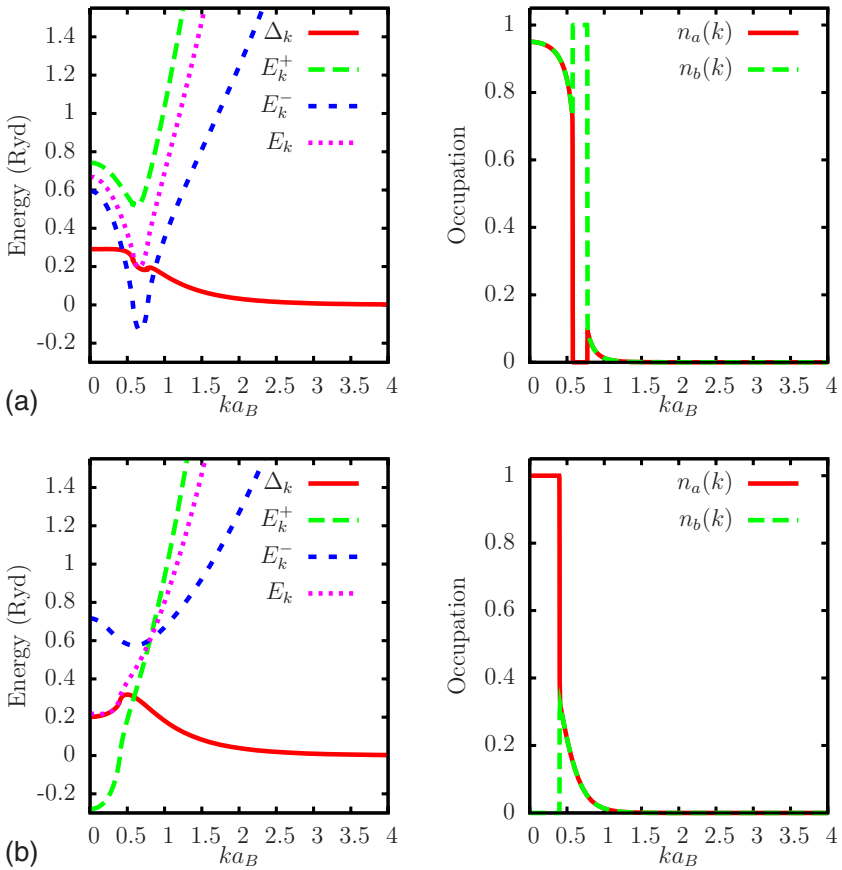

FIG. 1. (Color online) Gap function and quasiparticle energies with bare Coulomb interactions for $m_{e} / m_{h}=0.07 / 0.30$ and $d=a_{B}$ The upper panel shows a Sarma-2 phase at $r_{s}=3$ and $\alpha=-0.3$ with excess holes (heavy majority species). The lower panel shows a Sarma- 1 phase at $r_{s}=5$ and $\alpha=0.5$ with excess electrons (light majority species). Occupation numbers are shown on the right.

surface together with a condensate. The gap function is nonzero but there are gapless excitations.

\section{B. Quasiparticle properties}

In the following we present physical quantities in rydberg units, i.e., length is measured in effective (excitonic) Bohr radius $a_{B}=\frac{\hbar^{2} \varepsilon}{m e^{2}}$, momentum in $1 / a_{B}$, and energy in effective Rydberg $\left(\operatorname{Ryd}=\frac{\hbar^{2}}{2 m a_{B}^{2}}=\frac{e^{2}}{2 \varepsilon a_{B}}\right)$. The reduced mass $m$ is defined by $1 / m=1 / m_{a}+1 / m_{b}$, where $m_{a}=m_{e}$ and $m_{b}=m_{h}$ are the band mass of the electron and hole, respectively. In the numerical calculations we specialize to GaAs system parameters with mass ratio $m_{a} / m_{b}=0.07 / 0.30$ and background dielectric constant $\varepsilon=12.9$.

Representative solutions with one and two Fermi surfaces at $T=0$ are illustrated in Fig. 1 (bare Coulomb interaction) and Fig. 2 (screened interactions) for $d=a_{B}$. The figures show the gap function $\Delta_{\mathrm{k}}$, the quasiparticle energies $E_{\mathbf{k}}^{ \pm}$and their average $E_{\mathbf{k}}$ on the left panels, and the electron and hole occupation numbers $n_{a}(k), n_{b}(k)$ on the right panels. At $T$ $=0$ in the ground state, the quasiparticle levels with negative energy are occupied, positive-energy levels are empty. The two different type of excitation branches are split both due to different electron-hole mass and chemical-potential values. When one of the spectra crosses the zero-energy axis, population imbalance is created. If the negative-energy region includes the origin at $k=0$, the ground state has one Fermi surface, otherwise it has two. The two cases are denoted by S1 and S2, respectively. The top panel in each figure shows 

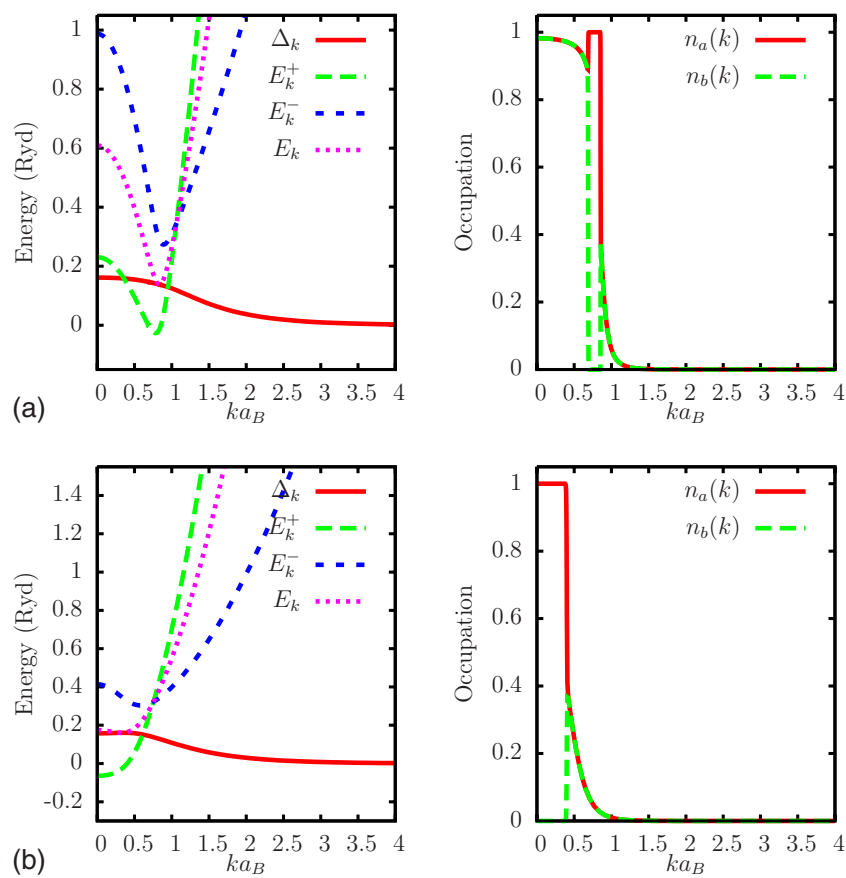

FIG. 2. (Color online) Gap function and quasiparticle energies with screened Coulomb interactions for $m_{e} / m_{h}=0.07 / 0.30$ and $d$ $=a_{B}$. The upper panel shows a Sarma-2 phase at $r_{s}=2.5$ and $\alpha$ $=0.2$ with excess electrons. The lower panel shows a Sarma-1 phase at $r_{s}=5$ and $\alpha=0.5$ with excess electrons. Occupation numbers are shown on the right. The gap function has less variation and the divergence in the derivative at the zero crossings disappears.

an S2 phase and the bottom panel shows an S1 phase. Since the quasiparticle energy branch is continuous the system still has gapless excitations. A close investigation of the gap function $\Delta_{\mathbf{k}}$ in the absence of screening (Fig. 1) shows that it has a cusp at the zero crossings of the quasiparticle energy, corresponding to a divergence in the derivative of $\Delta_{\mathbf{k}}$. This divergence has important consequences on the stability of the Sarma phase at $T=0$, as discussed below.

\section{Superfluid mass density, compressibility matrix, and the stability of the Sarma phase}

The "local" stability of the Sarma phase with respect to phases of the FFLO type is usually assessed by calculating the superfluid mass density (phase stiffness). ${ }^{20,27,53}$ This quantity should be positive in a stable state and a negative value is identified with an instability toward an FFLO phase. ${ }^{17,18,53}$ Clearly, the positivity of the superfluid mass density guarantees only that the Sarma phase is a local minimum of the energy with respect to fluctuations of the gap parameter associated with pairing of the FFLO type, and does not exclude the possibility that an FFLO phase with finite pair momentum can be a global minimum of the energy. When this happens, the local stability of the Sarma phase actually corresponds to metastability.

The superfluid mass density is given by ${ }^{27}$
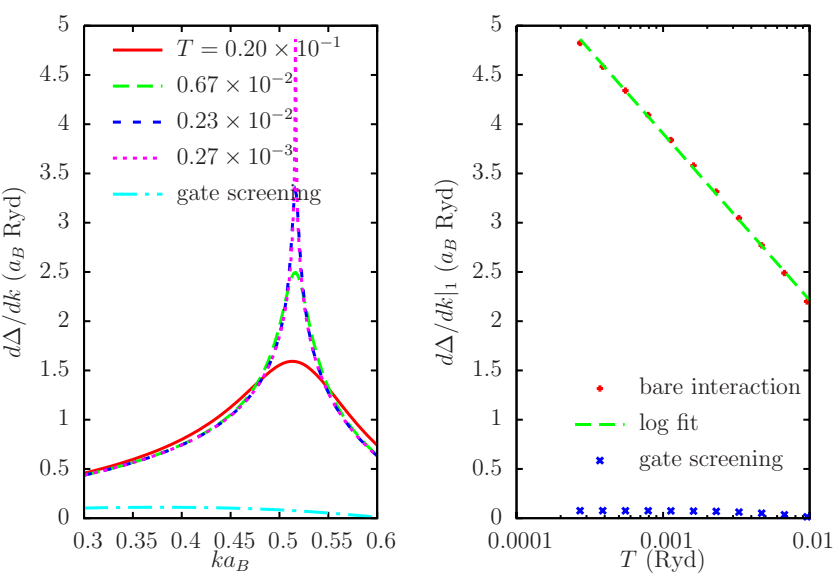

FIG. 3. (Color online) The derivative of the gap function $d \Delta_{\mathbf{k}} / d k$ as a function of $k$ at various temperatures with bare interlayer interactions, or at $T=0$ with gate screening (left). The value $d \Delta_{\mathbf{k}} /\left.d k\right|_{1}$ at the zero crossing of $E_{\mathbf{k}}^{+}$as a function of temperature $T$ with bare interlayer interactions or with gate screening (right). In both panels: $d=a_{B}, r_{s}=3, \alpha=0.3$, and $m_{e} / m_{h}=0.07 / 0.30$.

$$
\begin{aligned}
\rho_{s}= & m_{e} n_{e}+m_{h} n_{h}-\frac{\hbar^{2} \beta}{8 \pi} \int d k k^{3} \frac{1}{2}\left[\frac{1}{\cosh ^{2}\left(\beta E_{\mathbf{k}}^{+} / 2\right)}\right. \\
& \left.+\frac{1}{\cosh ^{2}\left(\beta E_{\mathbf{k}}^{-} / 2\right)}\right],
\end{aligned}
$$

where $\beta$ is the inverse temperature. At $T=0$ this expression can be written as ${ }^{53}$

$$
\rho_{s}=m_{e} n_{e}+m_{h} n_{h}-\frac{\hbar^{2}}{4 \pi} \sum_{j, \lambda} \frac{\left(k_{j}^{\lambda}\right)^{3}}{\left|\frac{d E_{k}^{\lambda}}{d k}\right|_{k=k_{j}^{\lambda}}},
$$

where $k_{j}^{\lambda}$ are the roots of $E_{k}^{\lambda}$ with $\lambda= \pm$. At zero temperature the last expression involves the derivative of $\Delta_{k}$ (through the derivative of $E_{k}^{ \pm}$) at the zero crossings of $E_{k}^{ \pm}$. As mentioned above, our calculations are carried out at nonzero but small temperature. We have found that this derivative diverges logarithmically as $T \rightarrow 0$. In particular, we have demonstrated analytically that for the bare Coulomb interaction one has

$$
\left|\frac{d \Delta_{\mathbf{k}}}{d k}\right|_{k=k^{*}} \approx \frac{e^{2}}{\pi \varepsilon} \frac{\Delta_{\mathbf{k}^{*}}}{2 E_{\mathbf{k}^{*}}}|\ln T| \text { as } T \rightarrow 0,
$$

where $k^{*}$ is the zero crossing point at $T=0$ as $k \rightarrow k^{*}$

$$
\left|\frac{d \Delta_{\mathbf{k}}}{d k}\right|_{T=0} \approx \frac{e^{2}}{\pi \varepsilon} \frac{\Delta_{\mathbf{k}^{*}}}{2 E_{\mathbf{k}^{*}}} \ln \left|k-k^{*}\right| \text { as } k \rightarrow k^{*},
$$

which we have also checked numerically (Fig. 3). This divergence is due to the simultaneous presence of the longrange Coulomb interaction, which is singular at $q=0$, and the discontinuity of the Fermi function at $T=0$. Finite temperature and/or screening effects, smear out these singularities thus removing the divergence. The presence of this divergence at $T=0$ was overlooked in the previous mean-field study of the imbalanced electron-hole bilayer system. ${ }^{53}$ As a matter of fact, making this divergence emerge from the nu- 
merical calculation requires the achievement of very low temperatures in the calculations and an extreme precision in the numerical integration.

The presence of this divergence is particularly meaningful for the analysis of the local stability of the Sarma phase at strictly $T=0$. The diverging derivative makes, in fact, the negative contribution to $\rho_{s}$ vanish, thus implying that for the unscreened Coulomb interaction the Sarma phase is always locally stable at $T=0$. This result is interesting as a matter of principle, as it offers an "extreme" example, where the argument by Forbes et l. $^{22}$ that mass ratio and momentum structure of the interactions should favor the stability of Sarma phase is completely effective. On the other hand we expect it to have few practical consequences, since the stability of the Sarma phase induced by this divergence is very fragile with respect to finite temperature and/or screening effects. As Fig. 3 clearly shows, quite small temperatures suffice to smear out the divergence in the derivative. Alternatively, the simple screened interaction makes the divergence to disappear even at $T=0$, as also shown in Fig. 3. As a result, in the presence of screening, the Sarma phase will indeed be locally unstable in certain regions of the $r_{s}-\alpha$ plane, as discussed in the next section.

The mechanical stability of the system with respect to phase separation requires the compressibility matrix $\partial \mu_{i} / \partial N_{j}$ to be positive definite. We have therefore calculated the compressibility matrix across our phase diagram to check also this stability. When the intralayer Coulomb interaction is neglected, the compressibility matrix develops negative eigenvalues across most of our phase diagram (restricting the stable region only to small values of $r_{s}$ ) in agreement with the findings of the recent work by Yamashita et al. ${ }^{57} \mathrm{How}-$ ever, as it was already argued in Ref. 53, this apparent dominant instability toward phase separation is an artifact occurring when the intralayer Coulomb repulsion is artificially excluded from the calculation. It should therefore not be taken seriously. In particular, we have verified explicitly that in our calculations with Coulomb intralayer repulsion, the Hartree term, which increases linearly with the distance $d_{G}$ between the metallic gates and the electron/hole layers, washes out completely phase separation from our phase diagram of Fig. 4 already for distances $d_{G}$ on the order of $5 a_{B}-10 a_{B}$, well below the typical gate-to-layer distances in current devices. We thus conclude that, contrary to what happens in cold atom systems, phase separation is not an issue in electron-hole bilayer systems.

\section{Phase diagram at $d=a_{B}$}

In this section we present the phase diagram resulting from the comparison of the energies of the Sarma and normal phases and from the stability analysis discussed in the previous section. We set the interlayer separation equal to one effective (excitonic) Bohr radius $d=a_{B}$. To make contact with previous literature, we present in Fig. 4 the phase diagram for progressively refined approximations corresponding to the inclusion in the calculations of: (i) bare interlayer interactions only, (ii) bare interlayer and intralayer interactions, (iii) screened interlayer interactions only, and (iv) screened interlayer and intralayer interactions.
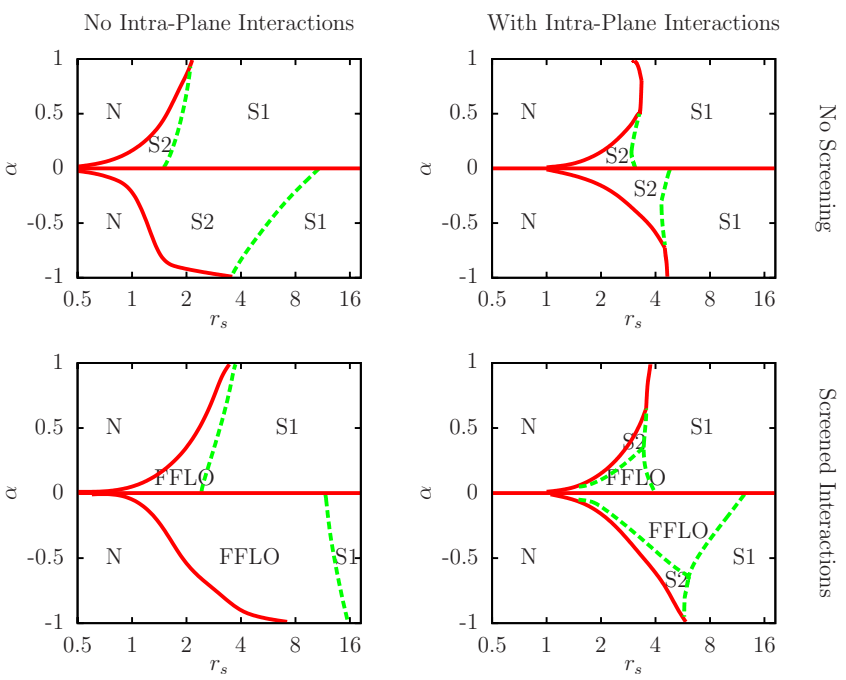

FIG. 4. (Color online) Phase diagram for interlayer separation $d=a_{B}$ with progressive refinement of the approximations. Superfluid (S1/S2)-normal (N) phase boundaries are shown with red solid lines. A negative superfluid mass density showing a local instability is assumed to be toward an FFLO phase. S1, S2, and FFLO boundaries are shown with green dashed lines. The four panels correspond to calculations including: bare interlayer interactions only (upper left), bare intralayer and interlayer interactions (upper right), gate screened interlayer interactions only (lower left) and gate screened intralayer and interlayer interactions (lower right). The $\alpha=0$ line is special in the phase diagram and corresponds to the BCS state with equal populations, which is always stable.

For bare interactions, the superfluid density is always positive and the Sarma phase is "locally" stable, due to the mechanism explained in Sec. III C. Therefore, in the top panel of Fig. 4 we do not show any FFLO phase, but our calculations do not rule out the possibility of a first-order transition to an FFLO phase with a finite FFLO modulation momentum $\mathbf{q}$ as found in Ref. 57. Two topologically distinct Sarma phases, Sarma-1 with one Fermi surface and Sarma-2 with two Fermi surfaces, are present in the phase diagrams. The effect of the intralayer repulsive interactions is to favor the normal phase with respect to the condensed phases, thus shifting to higher values of $r_{s}$ the boundary between normal and condensed phases (right panels). The two bottom panels of Fig. 4 presents the phase diagram when the gate screening is taken into account. With interlayer interactions only, the Sarma phase becomes unstable for a large portion of the phase diagram, especially with excess holes, i.e., $\alpha<0$ (bottom left panel). There is no stable S2 phase in this case. Switching on the intralayer interactions reduces the space occupied by the FFLO phase in our phase diagram and restores the S2 phase in some region of the phase diagram. This result is physically quite sensible, as the FFLO modulations of the gap parameter should be unavoidably accompanied by some modulations of the density in real space. In the presence of the Coulomb intralayer repulsion such density modulations are energetically expensive, thus hindering the FFLO phase with respect to the Sarma phase. ${ }^{58}$

A quite rich phase diagram is therefore obtained when both intralayer and screening effects are present. The pres- 
ence of locally stable Sarma phases confirms the expectation that isotropic translationally invariant gapless superfluid states can be stable with momentum-dependent interaction. ${ }^{22}$ We note in this context that in recent work, Sarma phases were found to be stable also in two-dimensional, two-band neutral Fermi systems. ${ }^{38,41}$

\section{SUMMARY}

We have studied a bilayer system of electron and hole layers spatially separated by an insulating barrier, where the electron and hole densities can be controlled independently and have analyzed $s$-wave pairing between electrons and holes as a function of average density and population difference using mean-field theory. By solving the relevant energy-gap equations we have compared the energy of the condensed phase called the Sarma phase with that of the normal state which is the sum of the electron and hole Fermiliquid energy described by the Hartree-Fock solution. We have included both interlayer and intralayer interactions generalizing earlier work which did not include in-plane interactions. ${ }^{53}$ In this way the phase boundary for the ground state is established in the density-population polarization $\left(r_{s}-\alpha\right)$ plane. The "local" stability of the Sarma phase was checked by calculating the superfluid mass density, whereas the stability with respect to phase separation was assessed by calculating the compressibility matrix. We have found that with bare Coulomb interactions the Sarma phase is always locally stable due to a peculiar momentum structure of the gap function originating from the singular infrared behavior of the Coulomb potential, and the simultaneous presence of a sharp Fermi surface at zero temperature.
Employing a simple model of screening which introduces an infrared cutoff in the Coulomb interaction, we have found that some regions in the phase space become unstable. We interpret this as an instability toward an FFLO phase. Together with intralayer interactions, the phase diagram in the crossover regime from the weakly interacting high-density BCS limit to the strongly interacting BEC of dilute excitons has room for various phases. The topologically different S1 and S2 Sarma phases and FFLO are present with the inclusion of screening and intralayer interactions. On the other hand, without any screening there is no instability toward FFLO and turning-off intralayer interactions the phase diagram does not show an S2 state. Currently, the experimental situation allows these systems to be realized. ${ }^{51,52,59}$ Quantitative comparison would require a more realistic model of screening, accounting for the condensed phase and finite width of the quantum wells, incorporating the disorder effects, and inclusion of spin degrees of freedom which may enter nontrivially when there are spin-dependent interactions such as spin-orbit coupling. With the renewed mean-field phase diagram at hand, it would also be interesting to perform QMC simulations to probe the predicted phases.

\section{ACKNOWLEDGMENTS}

This work is supported by TUBITAK (Grant No. 108T743), TUBA, European Union 7th Framework project UNAM-REGPOT (Grant No. 203953), and the Italian MUR under Contract No. PRIN-2007 "Ultracold Atoms and Novel Quantum Phases." A.L.S. thanks TUBITAK-BAYG and the hospitality of the Theoretical Physics Department of the University of Trieste during the time he spent there at an earlier stage of this work.
*Present address: Faculty of Engineering and Natural Sciences, Sabanci University, Tuzla, 34956 Istanbul, Turkey.

${ }^{1}$ M. Greiner, C. A. Regal, and D. S. Jin, Nature (London) 426, 537 (2003).

${ }^{2}$ S. Jochim, M. Bartenstein, A. Altmeyer, G. Hendl, S. Riedl, C. Chin, J. Hecker Denschlag, and R. Grimm, Science 302, 2101 (2003).

${ }^{3}$ M. W. Zwierlein, C. A. Stan, C. H. Schunck, S. M. F. Raupach, S. Gupta, Z. Hadzibabic, and W. Ketterle, Phys. Rev. Lett. 91, 250401 (2003).

${ }^{4}$ C. Chin, M. Bartenstein, A. Altmeyer, S. Riedl, S. Jochim, J. Hecker Denschlag, and R. Grimm, Science 305, 1128 (2004).

${ }^{5}$ C. A. Regal, M. Greiner, and D. S. Jin, Phys. Rev. Lett. 92, 040403 (2004).

${ }^{6}$ M. W. Zwierlein, J. R. Abo-Shaeer, A. Schirotzek, C. H. Schunck, and W. Ketterle, Nature (London) 435, 1047 (2005).

${ }^{7}$ M. W. Zwierlein, A. Schirotzek, C. H. Schunck, and W. Ketterle, Science 311, 492 (2006).

${ }^{8}$ G. B. Partridge, W. Li, R. I. Kamar, Y.-an Liao, and R. G. Hulet, Science 311, 503 (2006).

${ }^{9}$ C. H. Schunck, Y. Shin, A. Schirotzek, M. W. Zwierlein, and W. Ketterle, Science 316, 867 (2007).

${ }^{10}$ J. T. Stewart, J. P. Gaebler, and D. S. Jin, Nature (London) 454,
744 (2008).

${ }^{11}$ R. Jördens, N. Strohmaier, K. Günter, H. Moritz, and T. Esslinger, Nature (London) 455, 204 (2008).

${ }^{12}$ G.-B. Jo, Ye-Ryoung Lee, Jae-Hoon Choi, Caleb A. Christensen, Tony H. Kim, Joseph H. Thywissen, David E. Pritchard, Wolfgang Ketterle, Science 325, 1521 (2009).

${ }^{13}$ I. Bloch, J. Dalibard, and W. Zwerger, Rev. Mod. Phys. 80, 885 (2008).

${ }^{14}$ S. Giorgini, L. P. Pitaevskii, and S. Stringari, Rev. Mod. Phys. 80, 1215 (2008).

${ }^{15}$ C. A. R. Sá de Melo, Phys. Today 61 (10) 45 (2008).

${ }^{16}$ G. Sarma, J. Phys. Chem. Solids 24, 1029 (1963).

${ }^{17}$ P. Fulde and R. A. Ferrell, Phys. Rev. 135, A550 (1964).

${ }^{18}$ A. I. Larkin and Y. N. Ovchinnikov, Zh. Eksp. Teor. Fiz. 47, 1136 (1964) [Sov. Phys. JETP 20, 762 (1965)].

${ }^{19}$ W. V. Liu and F. Wilczek, Phys. Rev. Lett. 90, 047002 (2003).

${ }^{20}$ S.-T. Wu and S. Yip, Phys. Rev. A 67, 053603 (2003).

${ }^{21}$ P. F. Bedaque, H. Caldas, and G. Rupak, Phys. Rev. Lett. 91 247002 (2003)

${ }^{22}$ Michael McNeil Forbes, E. Gubankova, W. V. Liu, and F. Wilczek, Phys. Rev. Lett. 94, 017001 (2005).

${ }^{23}$ J. Carlson and S. Reddy, Phys. Rev. Lett. 95, 060401 (2005).

${ }^{24}$ D. E. Sheehy and L. Radzihovsky, Phys. Rev. Lett. 96, 060401 
(2006).

${ }^{25}$ F. Chevy, Phys. Rev. Lett. 96, 130401 (2006).

${ }^{26}$ P. Pieri and G. C. Strinati, Phys. Rev. Lett. 96, 150404 (2006).

${ }^{27}$ M. Iskin and C. A. R. Sá de Melo, Phys. Rev. Lett. 97, 100404 (2006).

${ }^{28}$ C.-H. Pao, Shin-Tza Wu, and S.-K. Yip, Phys. Rev. B 73, 132506 (2006).

${ }^{29}$ C.-C. Chien, Q. Chen, Y. He, and K. Levin, Phys. Rev. Lett. 97, 090402 (2006).

${ }^{30}$ K. B. Gubbels, M. W. J. Romans, and H. T. C. Stoof, Phys. Rev. Lett. 97, 210402 (2006).

${ }^{31}$ P. Nikolić and S. Sachdev, Phys. Rev. A 75, 033608 (2007).

${ }^{32}$ M. M. Parish, F. M. Marchetti, A. Lamacraft, and B. D. Simons, Nat. Phys. 3, 124 (2007).

${ }^{33}$ M. Iskin and C. A. R. Sá de Melo, Phys. Rev. A 76, 013601 (2007).

${ }^{34}$ S. Pilati and S. Giorgini, Phys. Rev. Lett. 100, 030401 (2008).

${ }^{35}$ G. J. Conduit, P. H. Conlon, and B. D. Simons, Phys. Rev. A 77, 053617 (2008).

${ }^{36}$ R. Sharma and S. Reddy, Phys. Rev. A 78, 063609 (2008).

${ }^{37}$ G. Bertaina and S. Giorgini, Phys. Rev. A 79, 013616 (2009).

${ }^{38}$ L. He and P. Zhuang, Phys. Rev. B 79, 024511 (2009).

${ }^{39}$ Y. Chen, Z. D. Wang, F. C. Zhang, and C. S. Ting, Phys. Rev. B 79, 054512 (2009).

${ }^{40}$ S. K. Baur, S. Basu, T. N. De Silva, and E. J. Mueller, Phys. Rev. A 79, 063628 (2009).

${ }^{41}$ T. N. De Silva, J. Phys. B 42, 165301 (2009).

${ }^{42}$ Y. Shin, M. W. Zwierlein, C. H. Schunck, A. Schirotzek, and W. Ketterle, Phys. Rev. Lett. 97, 030401 (2006).

${ }^{43}$ G. B. Partridge, W. Li, Y. A. Liao, R. G. Hulet, M. Haque, and H. T. C. Stoof, Phys. Rev. Lett. 97, 190407 (2006).

${ }^{44}$ Y. E. Lozovik and V. Yudson, Zh. Eksp. Teor. Fiz. Pis'ma Red. 22, 556 (1975) [JETP Lett. 22, 274 (1975)]; Zh. Eksp. Teor. Fiz. 71, 738 (1976) [Sov. Phys. JETP 44, 389 (1976)].
${ }^{45}$ S. I. Shevchenko, Fiz. Nizk. Temp. 2, 505 (1976) [Sov. J. Low Temp. Phys. 2, 251 (1976)].

${ }^{46}$ L. V. Butov, A. C. Gossard, and D. S. Chemla, Nature (London) 418, 751 (2002); A. T. Hammack, M. Griswold, L. V. Butov, L. E. Smallwood, A. L. Ivanov, and A. C. Gossard, Phys. Rev. Lett. 96, 227402 (2006); S. Yang, A. T. Hammack, M. M. Fogler, L. V. Butov, and A. C. Gossard, ibid. 97, 187402 (2006).

${ }^{47}$ X. Zhu, M. S. Hybertsen, and P. B. Littlewood, Phys. Rev. B 54, 13575 (1996).

${ }^{48}$ S. De Palo, F. Rapisarda, and G. Senatore, Phys. Rev. Lett. 88, 206401 (2002); G. Senatore and S. De Palo, Contrib. Plasma Phys. 43, 363 (2003).

${ }^{49}$ J. A. Keogh, Appl. Phys. Lett. 87, 202104 (2005).

${ }^{50}$ J. A. Seamons, Appl. Phys. Lett. 90, 052103 (2007).

${ }^{51}$ A. F. Croxall, K. Das Gupta, C. A. Nicoll, M. Thangaraj, H. E. Beere, I. Farrer, D. A. Ritchie, and M. Pepper, Phys. Rev. Lett. 101, 246801 (2008).

${ }^{52}$ J. A. Seamons, C. P. Morath, J. L. Reno, and M. P. Lilly, Phys. Rev. Lett. 102, 026804 (2009).

${ }^{53}$ P. Pieri, D. Neilson, and G. C. Strinati, Phys. Rev. B 75, 113301 (2007).

${ }^{54}$ Z. W. Gortel and L. Świerkowski, Surf. Sci. 361-362, 146 (1996).

${ }^{55}$ L. H. Ho, A. P. Micolich, A. R. Hamilton, and O. P. Sushkov, Phys. Rev. B 80, 155412 (2009).

${ }^{56}$ For QMC applications this state can be written as a Slater determinant in real space.

${ }^{57}$ K. Yamashita, K. Asano, and T. Ohashi, arXiv:0908.2492 (unpublished).

${ }^{58}$ For the same reason we expect that inclusion of intralayer interaction could strongly affect the mean-field phase diagram of Ref. 57.

${ }^{59}$ A. F. Croxall, K. Das Gupta, C. A. Nicoll, H. E. Beere, I. Farrer, D. A. Ritchie, and M. Pepper, Phys. Rev. B 80, 125323 (2009). 